\section{NOVA TELLVS}

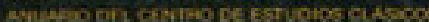

Nova Tellus

ISSN: 0185-3058

novatelu@servidor.unam.mx

Centro de Estudios Clásicos

México

LEJAVITZER L., Amalia

Algunas recetas médicas en el De re coquinaria de Apicio

Nova Tellus, vol. 24, núm. 1, 2006, pp. 123-139

Centro de Estudios Clásicos

Distrito Federal, México

Disponible en: http://www.redalyc.org/articulo.oa?id=59114742006

- Cómo citar el artículo

- Número completo

- Más información del artículo

- Página de la revista en redalyc.org

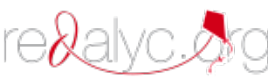

Sistema de Información Científica

Red de Revistas Científicas de América Latina, el Caribe, España y Portugal

Proyecto académico sin fines de lucro, desarrollado bajo la iniciativa de acceso abierto 


\title{
Algunas recetas médicas en el De re coquinaria de Apicio
}

\author{
Amalia LeJAVITZER L. \\ Universidad Nacional Autónoma de México \\ alejavitzer@gmail.com
}

RESUMEN: En este artículo se estudian varias de las casi treinta recetas médicas contenidas en De re coquinaria de Apicio; se propone una posible clasificación de ellas, y se muestran ciertas semejanzas y diferencias entre las recetas apicianas y algunos preceptos expresados por Galeno en De alimentorum facultatibus y en De ptisana.

$$
* * *
$$

ABstRACT: This article studies several of the almost thirty medical recipes contained in Apicius' De re coquinaria. It proposes a tentative classification of them, and also reveals certain similarities and differences between Apicius' prescriptions and some precepts that galen mentions in his De alimentorum facultatibus and De ptisana.

Palabras Clave: alimentación, Apicio, coquinaria, medicina, recetas.

RECEPCIÓN: 18 de enero de 2006.

ACEPTACIÓn: 17 de febrero de 2006. 



\title{
Algunas recetas médicas en el De re coquinaria de Apicio
}

\author{
Amalia LeJAVITZER L.
}

Sin alimento no podemos vivir, ni los sanos ni los enfermos.

Galeno, VI, $453 \mathrm{~K}$

Hoy en día, nadie desconoce la estrecha relación que guarda la alimentación con la salud. Los antiguos griegos y romanos tampoco la ignoraron, como resulta evidente a partir de las palabras de Galeno que acabo de citar. Más aún, la dieta tuvo un papel determinante tanto en el ámbito de la alimentación como en el de la medicina, e incluso en el de la filosofía de la antigüedad clásica.

Pitágoras, Platón, Aristóteles, Plutarco, Porfirio y Jámblico, entre otros filósofos, se preocuparon por precisar qué alimentos debían ingerirse para mantener la salud y la pureza del organismo en su conjunto, física y espiritualmente hablando. ${ }^{1}$ Muy conocidas son, por ejemplo, la prohibición de comer habas entre los pitagóricos, ${ }^{2}$ o la apología del vegetarianismo de Porfirio, ${ }^{3}$ con lo que la observancia de un régimen alimenticio concernía más a la esfera de lo moral, y a veces de lo sobrenatural, que a la de lo estrictamente alimentario.

\footnotetext{
${ }^{1}$ García Gual, pp. 43-67.

${ }^{2}$ Respecto a la prohibición de comer habas, cf. Empédocles (fr. 141 DielsKranz), Aristoxeno (fr. 25 Wehrli), Calímaco (fr. 553 Pfeiffer), Cicerón (Div., I, xxx, 62) y Aulo Gelio (N. A., IV, xi).

${ }^{3}$ García Gual, pp. 59-66.
} 
Ahora bien, la única obra que conservamos en materia de alimentación del imperio romano es el tratado conocido como De re coquinaria, atribuido a Apicio. Este compendio de casi quinientas recetas, data del siglo cuarto de nuestra era, y recoge el saber alimentario de su tiempo y de tiempos anteriores. Sobre esta obra mucho se ha dicho tanto acerca de su autor, de la época de su composición, de la lengua en la que está escrita, como de la sofisticación de sus recetas, y de su posible (o imposible) adaptación a la cocina contemporánea, hasta de la degradación moral de las costumbres del tardo imperio que ella refleja; pero, hasta donde sé, poco se ha estudiado la vinculación de varias de sus recetas con la medicina, en general, y con la dietética, en particular: sobre este aspecto trataré en estas páginas.

En primer lugar, es necesario aclarar que, en la antigüedad,

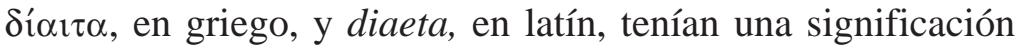
mucho más amplia de la que hoy en día damos a "dieta", su equivalente palabra castellana, pues tanto el término griego como el latino se referían no sólo a qué alimentos comer, sino a cómo vivir, a cómo llevar una vida saludable en cuerpo y alma, tal como sentencia el célebre verso de Juvenal, mens sana in corpore sano. ${ }^{4}$

La dieta, en la antigüedad, implicaba llevar un género de vida saludable, que incluyera, por supuesto, una alimentación sana, pero también ejercicio frecuente, periodos de reposo, purgas habituales y baños. ${ }^{5} \mathrm{Y}$ su papel en la medicina antigua era, ante todo, el de conservar la salud y el de prevenir la enfermedad, ${ }^{6}$ causada por la alteración del equilibrio interno. La dieta, pues, tenía la función de restaurar el equilibrio fracturado, o de impedir, mediante su cumplimiento, que dicho equilibrio se quebrantara.

\footnotetext{
${ }^{4}$ Juv., X, 356.

${ }^{5}$ Mazzini, v. 2, p. 350.

${ }^{6}$ Idem.
} 
Para Hipócrates, "la salud es el resultado del equilibrio entre aquello que se ingiere y aquello que el cuerpo consume". ${ }^{7}$ Los tratados Sobre la dieta, Sobre la dieta sana, Sobre la dieta en las enfermedades agudas, Sobre el uso de los líquidos, entre otras obras que se incluyen en el Corpus Hippocraticum, revelan la importancia que el médico de Cos otorgó a la dieta. Más aún, la dieta constituye una de las partes fundamentales de la terapia en la antigüedad, pues, como sintetiza Celso, la medicina "se divide en tres partes: una que cura por medio de la dieta; otra, por medio de medicamentos, y la tercera que lo hace por medio de la mano. Los griegos llamaron a la primera, dietética; a la segunda, farmacéutica, y a la tercera, cirugía".

Pero sobre todo fue Galeno, nacido bajo el reino de Adriano, en Pérgamo, considerado "el último de lo grandes médicos creadores de la Antigüedad, el segundo 'padre fundador' de la medicina antigua después de Hipócrates", 9 quien situó a la dieta en un lugar prominente dentro del arte médica: para él "la dietética no sólo es el fundamento tradicional de la terapéutica, desde el punto de vista puramente doctrinal, sino una experiencia vital básica". ${ }^{10}$

Galeno conservó la teoría hipocrática de los cuatro humores, que concebía el cuerpo humano compuesto por la sangre, caracterizada por la cualidad de lo cálido; la flema, caracterizada por lo frío; la bilis amarilla, por lo húmedo, y la bilis negra, por lo seco. ${ }^{11}$ Solamente mediante el equilibrio ideal de los cuatro humores se podía alcanzar y conservar la salud. ${ }^{12}$

\footnotetext{
${ }^{7}$ Gil Sotres, p. 257.

${ }^{8}$ Cels., proem., 9: Iisdemque temporibus in tres partes medicina diducta est, ut una esset quae uictu, altera quae medicamentis, tertia quae manu mederetur. Pri-

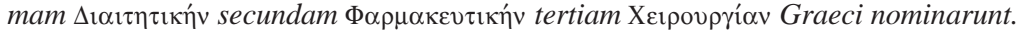

${ }^{9}$ Gourevitch, p. 112.

${ }^{10}$ García Ballester, p. 231.

${ }^{11}$ A su vez, cada uno de estos humores se relacionaba con un órgano del cuerpo: con el corazón, con el cerebro, con el hígado y con el bazo, respectivamente, cf. Gourevitch, p. 115.

${ }^{12}$ Gil Sotrés, p. 259.
} 
En consecuencia, el médico debía ser capaz de conocer

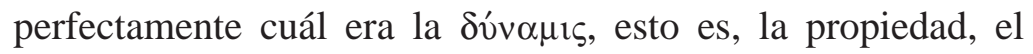
poder o la facultad de cada alimento, para que, a través de este conocimiento, pudiera indicarlo con total exactitud a sus pacientes, y utilizarlo de la mejor manera posible para preservar ese equilibrio corporal o ayudar a recobrarlo cuando se ha perdido. ${ }^{13}$ En su obra De alimentorum facultatibus, Acerca de las propiedades de los alimentos, una de las cinco referidas a la dietética, ${ }^{14}$ Galeno clasifica los alimentos según sus facul-

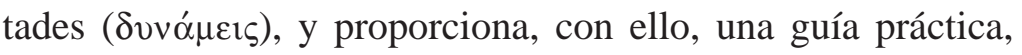
tanto para el médico como para el lego, a fin de prescribir o de seguir una dieta beneficiosa, y determinar qué comida o bebida satisfacía las necesidades humorales de cada individuo.

Veamos, ahora, algunas de las recetas médicas que se encuentran en De re coquinaria, para esclarecer su relación con la medicina de la antigüedad, y especialmente con el escrito galénico De alimentorum facultatibus.

En la obra de Apicio, en mi opinión, se pueden distinguir tres clases de recetas para preparar remedios: la primera integrada por las recetas que tienen una finalidad puramente medicinal; la segunda, por aquellas que, si bien fueron concebidas como preparaciones culinarias, tienen una prescripción médica como indicación accesoria; por último, la tercera está formada por recetas que no tienen una utilidad terapéutica explícita, pero que, sin embargo, por tradición se les atribuía. En total, entre los tres grupos, suman cerca de treinta las recetas de contenido médico en el De re coquinaria.

En la mayoría de las recetas de la primera clase, la prescripción médica se expresa desde el mismo título o en el del

\footnotetext{
${ }^{13}$ Volpe Cacciatore, p. 93.

${ }^{14}$ Las otras cuatro obras son: De bonis malisque sucis; De victu attenuante; De ptisana, y De sanitate tuenda.
} 
capítulo que las agrupa. Tal es el caso de la preparación que se intitula "oxígaro para la digestión" (oxygarum digestibilem), bebida hecha a base de garo ${ }^{15} \mathrm{y}$ vinagre, empleada como tónico reconstituyente y como remedio para mejorar la digestión y la motilidad del vientre. ${ }^{16}$

Otro ejemplo lo constituye el segundo capítulo del libro tercero, "Del huerto" (Cepuros, en latín), donde se incluyen cinco recetas agrupadas bajo el lema de Pulmentarium ad ventrem, esto es, "Potajes para el estómago". Como resulta evidente, todas estas recetas son remedios para aliviar los problemas del vientre. A continuación, transcribo una de ellas:

Otra [Receta] para el estómago: Se limpian unos manojos de acelga, sin lavarlos. Espolvoreas nitro en medio de ellos y atas cada uno de los manojos. Los pones en agua. Cuando se hayan cocido, los colocas en un recipiente junto con vino paso o con careno, ${ }^{17}$ sobre ellos espolvoreas pimienta y comino, y viertes un poco de aceite. Cuando haya hervido, mueles polipodio y trozos de nuez junto con garo; se vierte en un recipiente que esté hirviendo, se cubre. Se retira de inmediato y se emplea. ${ }^{18}$

Aquí, cabe hacer notar que la naturaleza medicinal de esta preparación es obvia no sólo por el lema del capítulo donde se inserta o por el propio título de la receta, cuyo ad ventrem

\footnotetext{
${ }^{15}$ El garo se elaboraba a partir de las entrañas de pescado (escombro o caba1la), especialmente de los intestinos, maceradas en sal y expuestas a una fuente de calor - la irradiación solar o el fuego-, hasta que los ingredientes se descomponían por completo, y la substancia quedaba reducida a una materia viscosa, de olor penetrante.

16 Apic., I, Xx, 1 (39). Sobre las propiedades medicinales del garo y sus compuestos he tratado con más detalle en Lejavitzer, pp. 115-128.

${ }^{17}$ El carenum (al igual que el defritum y la sapa) pertenece a los llamados vina cocta; este vino cocido, sumamente dulce y espeso, debido a la cocción a que era sometido, por medio de la cual se reducía el nivel de líquido y se concentraba la fructosa propia del mosto, en general, se empleaba exclusivamente en la cocina y en la medicina.

${ }^{18}$ Apic., III, II, 3 (69), ALITER AD VENTREM: Facies betaciorum fasces detergi, ne laves. In eorum medium nitrum asparges et adligas singulos fasces. Mittes in
} 
resulta muy elocuente por sí mismo, sino también por sus ingredientes:

1. La acelga (beta), según Galeno, tiene poder laxante y sirve para la evacuación de los intestinos; además, en su opinión, resulta un buen remedio ( $\alpha \gamma \alpha \theta$ òv pó $\rho \mu \alpha \kappa o v)$ si se come con mostaza o con vinagre. ${ }^{19}$ Aunque en la receta antes citada no aparece ninguno de estos dos condimentos, llama la atención que en el capítulo dedicado a la acelga, también del libro tercero del De re coquinaria, Apicio insiste en estos dos últimos ingredientes, pues afirma que "las acelgas hervidas: quedan bien con mostaza, un poco de aceite y vinagre". ${ }^{20}$

2. El polipodio (también llamado filicula, en latín) ${ }^{21}$ constituye un purgante, de uso exclusivo para la medicina, ${ }^{22}$ pues no hay evidencias de que la culinaria se haya servido de él; de hecho, en De re coquinaria aparece mencionado en sólo dos ocasiones: en ésta y en la receta precedente del capítulo de "Potajes para el estómago".

3. Las nueces, que aparecen molidas junto con el polipodio, para Galeno son muy útiles, pues son nutritivas y baratas, además de resultar benéficas para el estómago, cuando se comen junto con higos secos; en especial, ayudan a aligerar los intestinos, si se consumen junto con garo, ${ }^{23}$ tal y como se indica en la receta.

En algunas ocasiones, la noticia terapéutica forma parte del cuerpo de la receta, como en ésta de sales conditos ad multa,

aquam. Cum coxeris, condies patinam, cum eadem passum uel caroenum et cuminum et <piper> super asparges et oleum modicum. Vbi ferbuerit, polipodium et frusta nucum cum liquamine teres, feruentem patinam fundes, cooperies. Statim depones et uteris.

${ }^{19}$ Gal., VI, 629-630 K.

${ }^{20}$ Apic., III, XI, 2 (98), Aliter betas ELIXAS: Ex sinapi, oleo modico et aceto bene inferuntur.

${ }^{21}$ Pl., N. H., XXVI, 58: Polypodi, quam nostri f<e >liculam vocant.

${ }^{22}$ Col., VI, xxvii, 11; Cel., II, 12; Pl., N. H., XXVI, 105; 122; XXIX, 80.

${ }^{23}$ Gal., VI, 610-611 K. 
esto es, "sales condimentadas para muchos usos", cuya preparación comienza con el siguiente consejo:

Las sales condimentadas sirven para la digestión, para mover el vientre, e impiden que se generen toda clase de enfermedades y la peste y toda clase de resfriados $[\ldots]^{24}$

En otras, la recomendación médica se mezcla con lo que, en primera instancia, podría considerarse como una mera superstición:

Ortigas. Cuando el sol se encuentre en Aries, toma, si quieres, ortiga hembra contra cualquier enfermedad. ${ }^{25}$

No obstante, si tomamos en cuenta que el médico de Pérgamo "determinó los efectos de cada tipo de alimento, la estación del año, días y horas apropiados para su ingestión", ${ }^{26}$ la recomendación sole in ariete positio, cobra un nuevo significado, y lo que a simple vista parecía una superstición, puede volverse una explicación racional de precisión científica. Probablemente, en primavera la ortiga alcanzara mayor vigor en cuanto a sus propiedades ( $\delta v v \alpha ́ \mu \varepsilon ı \varsigma), y$, por lo tanto, acaso fuera más eficaz como remedio para aflojar el vientre, según señala Galeno, quien además afirma que constituye un alimento útil, aunque aclara que nadie la come, a menos de que se encuentre en un caso de hambre extrema, ${ }^{27}$ con lo cual queda de manifiesto, una vez más, que la finalidad de la receta apiciana era estrictamente medicinal.

\footnotetext{
${ }^{24}$ Apic., I, XIII (29), SALES CONDITOS AD MVLTA: Sales conditos ad digestionem, ad uentrem mouendum, et omnes morbos et pestilentiam et omnia frigora prohibent generari.

25 Apic., III, XVII (108), VRTICAE: Vrticam feminam, sole in ariete positio, aduersus aegritudinem sumes, si uoles.

${ }^{26}$ García Ballester, p. 232.

${ }^{27}$ Gal., VI, 639 K.
} 
La segunda clase de recetas comprende, como ya mencioné, aquellas que, si bien están escritas como preparaciones culinarias, por extensión también pueden ser utilizadas como medicamentos; por ejemplo, la receta para "conservar las uvas por mucho tiempo", donde, después de explicar los pasos para su conservación, se añade: "También esta misma agua se puede dar a los enfermos en lugar de hidromiel". ${ }^{28} \mathrm{O}$ esta otra, para preparar una "salmuera de pescado sin pescado":

Se muele tanto comino cuanto puedas tomar con cinco dedos, la mitad de esa cantidad de pimienta, y una cabeza de ajo limpia, se vierte garo y se rocía un poco de aceite. Esto recompone en gran manera el estómago enfermo y favorece la digestión. ${ }^{29}$

Consideremos, ahora, algunas recetas de la tercera clase, las cuales, aunque no se expresara, poseían un valor medicinal ampliamente reconocido, como la concerniente a los bulbos, que cito a continuación:

Se toman bulbos hervidos y exprimidos en una olla. Pones timo, orégano, miel, vinagre, defrito, ${ }^{30}$ dátil, garo, un poco de aceite. Se espolvorea pimienta y se lleva a la mesa.

[Varrón dijo: "Acerca de los bulbos: se sirven en agua, para quienes buscan la puerta del Amor, o en la cena, para las legí-

\footnotetext{
${ }^{28}$ Apic., I, XII, 1 (18), VVAE VT DIV SERVENTVR: Accipies uuas de uite inlaesas, et aquam pluuialem ad tertias decoques, et mittis in uas in quo et uuas mittis. Vas picari et gipsari facies, et in locum frigidum ubi soli accessum non habet reponi facies et, quando uolueris, uuas uirides inuenies. Et ipsam aquam pro idromelli aegris dabis. Et si in ordeo obruas, inlaesas inuenies.

${ }^{29}$ Apic., IX, XIII, 3 (432), ALITER SALSVM SINE SALSO: Cuminum tantum quantum quinque digitis tollis, piperis ad dimidium eius et unam spicam alei purgatam teres, liquamen superfundes, oleum modice superstillabis. Hoc aegrum stomachum ualde reficit et digestionem facit.

${ }^{30} \mathrm{El}$ defritum es una variedad de los vina cocta, es decir, de aquellos caldos en los cuales se reducía el líquido, a la mitad o más, mediante la cocción, obteniendo así un vino sumamente concentrado en azúcar; posteriormente, la palabra defritum por antonomasia pasó a designar todas las clases de vinos cocidos, cf. André, L'alimentation..., p. 164.
} 
timas nupcias, pero con piñones o con jugo de oruga y pimienta".] ${ }^{31}$

Más allá de la referencia a Varrón —imposible de localizar, y a juicio de muchos editores, ${ }^{32}$ una nota marginal que escribió algún lector, y que, después, en las copias sucesivas del manuscrito, fue incorporada al cuerpo de la receta-, estas líneas, en una primera lectura, parecieran asemejarse a una poción mágica o a una especie de conjuro amoroso. Sin embargo, encuentran su fundamento en el valor afrodisíaco que tanto la sabiduría popular como la medicina tradicional atribuyó a estas raíces. Un dístico de Marcial se hace eco de estas creencias: "Cuando tu esposa esté anciana, y muerto, tu miembro, con ninguna otra cosa, sino con bulbos, puedes quedar satisfecho". ${ }^{33}$ Mientras que Plinio afirma que los provenientes de Megara constituían un poderoso afrodisíaco ${ }^{34}$ por su parte, Galeno sostiene que los hombres que los han comido en abundancia han sentido de manera evidente un incremento en la producción de semen, y un aumento de su impulso sexual. ${ }^{35}$

Ahora bien, el ejemplo más notable de esta clase está representado por las recetas referidas a las ptisanae, las cuales constituían remedios eficaces, según la tradición médica y las costumbres populares; de hecho, Plinio afirma que el procedimiento para preparar las tisanas es por todos conocido, volga-

\footnotetext{
31 Apic., VII, XIV, 3 (307), ALITER: Bulbos elixos in pultarium pressos. Mittis timum, origanum, mel, acetum, defritum, careotam, liquamen, oleum modice. Piper aspargis et inferes.

[Varro: "Si quid de bulbis, dixi: in aquam, qui Veneris ostium quaerunt, deinde, ut legitimis nuptiis, in cena ponuntur, sed et cum nucleis pineis aut cum erucae suco et pipere".]

32 De esta opinión son Milham y André, 1987.

${ }^{33}$ Mart., XIII, 34: BVLbI. Cum sit anus coniunx et sint tibi mortua membra, I nil aliud bulbis quam satur esse potes.

${ }^{34} \mathrm{Pl} .$, N. H., XX, 105: venerem maxime Megarici stimulant.

${ }^{35} \mathrm{Gal} ., \mathrm{VI}, 653 \mathrm{~K}$.
} 
ta ratio est. ${ }^{36}$ En la obra de Apicio, aparecen cuatro recetas para preparar estas decocciones de granos, usualmente hechas a base de cebada, pero también de arroz ${ }^{37}$ o de trigo, ${ }^{38}$ y adicionadas con hierbas aromáticas y vinagre.

La eficacia terapéutica de las tisanas fue ampliamente reconocida por Galeno, quien, en su tratado De ptisana, explica sus propiedades y su adecuada preparación. Allí indica cómo cocinarla: previamente, se pone a remojar la cebada cruda; luego, se frota entre las manos para remover la cáscara y dejarla limpia (si se quiere un mayor poder laxante, se hierve con todo y cáscara); después, se hierve: primero, sobre fuego fuerte; más tarde, sobre fuego suave, hasta que se haga líquida. Entre sus propiedades, Galeno destaca que la tisana es una bebida hidratante, muy nutritiva, y de fácil digestión y asimilación, además señala su doble cualidad: la de ser un alimento grato al paladar y la de ser un remedio sumamente provechoso. ${ }^{39}$

Comparemos, ahora, una de las recetas conservadas en $D e$ re coquinaria:

Mientras la lavas, frotas la cebada, la cual se puso en remojo desde el día anterior. Se coloca sobre fuego fuerte. Cuando haya hervido, agregas bastante aceite, un manojo pequeño de anís, cebolla seca, ajedrea y un codillo de cerdo para que allí se cocine junto al jugo. Se agrega cilantro fresco y sal, triturados al mismo tiempo, y se hace que hierva. Cuando haya hervido bien, se retira el manojo y se pasa a otro cazo, para que así no se pegue al fondo a causa del calor. Se revuelve bien y se cuela en un cazo sobre el codillo. Se muele pimienta, legústico, ${ }^{40}$ un poco

\footnotetext{
${ }^{36} \mathrm{Pl} .$, N. H., XVIII, 75: tisanae conficiendae volgata ratio est.

${ }^{37}$ Pl., N. H., XVIII, 71; Hor., Sat., II, III, 155.

${ }^{38} \mathrm{Pl}$., N. H., XXII, 136.

${ }^{39}$ Gal., VI, 816-831 K.

${ }^{40}$ El legústico, ligusticum (Levisticum officinale Koch.) es una planta aromática de la familia de las umbelíferas, originaria de la cuenca del Mediterráneo,
} 
de poleo $^{41}$ seco, comino, laserpicio ${ }^{42}$ frito. Por encima se vierte miel, vinagre, defrito, garo. Viertes en el cazo, pero de modo que el codillo quede bien cubierto. Se hace que hierva sobre fuego lento. ${ }^{43}$

Ciertamente, la receta apiciana resulta mucho más elaborada y condimentada que la prescripción galénica; incluso reproduce lo que, a juicio del médico, constituye una manera equivocada, aunque habitual, de prepararla, pues nuestra receta agrega comino, miel, vinagre y vino. ${ }^{44}$ Sin embargo, en otros aspectos, sigue con exactitud las indicaciones expresadas por Galeno, y se aparta del uso común, pero erróneo, de moler la

también conocida como levístico, perejil silvestre, apio de monte o apio de montaña; se utilizan las semillas, la raíz y las hojas: las primeras, molidas, sustituyen a la pimienta; las últimas, a las del apio, y, desde la antigüedad, ha sido muy apreciada por sus muchas propiedades medicinales, pues activa la función de los riñones y la función del corazón, además, regula la circulación, por lo que es indicada en casos de retención de líquidos, trastornos menstruales, e incluso de arritmia cardiaca.

${ }^{41} \mathrm{El}$ poleo, puleium o pulegium (Mentha pulegium L.) es una hierba de olor agradable, pertenece al género de la menta, crece en lugares húmedos, especialmente a orillas de arroyos y manantiales, y se emplea como infusión para aliviar los problemas estomacales. Los antiguos solían conservarlo seco o en un frasco con vinagre.

${ }^{42}$ El laserpicio es una planta herbácea, conocida por los romanos bajo diferentes términos: silphium, laserpicium, lasar, sirpe; se le cree originaria del norte de África, de donde desapareció hacia la mitad del primer siglo de nuestra era, pero se siguió cultivando en Oriente (Persia, Siria y Armenia); se empleaba la semilla, la raíz, el tallo, y el jugo de ellos obtenido, como diurético y tónico digestivo.

43 Apic., IV, IV, 1 (173), TISANAM SIC FACIES: Tisanam lauando fricas, quam ante diem infundes. Impones supra ignem calidum. Cum bullierit, mittes olei satis et aneti modicum fasciculum, cepam siccam, satureiam et coloefium, ut ibi coquantur propter sucum. Mittes coriandrum uiridem et sale simul tritum et facies ut ferueat. Cum bene ferbuerit, tolles fasciculum et transferes in alterum caccabum tisanam sic ne fundum tangat propter combusturam. Lias et colas in caccabulo supra acronem coloefium. Teres piper, ligusticum, pulei aridi modicum, cuminum et sil frictum, suffundis 〈mel〉, acetum, defritum, liquamen, refundis in caccabum, sed coloefium acronem ut bene tegatur. Facias ut ferueat super ignem lentum.

${ }^{44}$ Gal., VI, $821 \mathrm{~K}$. 
cebada en el mortero y de cocinarla rápidamente. En la prescripción del De re coquinaria, en cambio, se pone a remojar la cebada con antelación; después se frota, quizá entre las manos, ya que no se menciona el empleo del mortero, y por último, se cocina lentamente, primero a fuego fuerte, luego a fuego suave.

En De re coquinaria aparece otra receta de tisana, en la cual se combina el grano de cebada con otras leguminosas, como garbanzo, chícharo y lenteja:

Remojas garbanzo, lenteja, chícharo. Se quita la cáscara a la cebada y se hierve con las leguminosas. Cuando haya hervido bien, agregas abundante aceite y se corta, todo muy fresco, puerro, cilantro, anís, hinojo, acelga, malva, col tierna. Estos vegetales cortados finamente se agregan al cazo. Hierves las coles y mueles bastante semilla de hinojo, orégano, laserpicio, legústico. Después de que se haya molido, templas con garo, y viertes por encima de las leguminosas, y revuelves. Por encima se cortan pequeños trozos de coles. ${ }^{45}$

Nuevamente podemos encontrar un antecedente de esta receta, en el médico de Pérgamo, ${ }^{46}$ quien recomienda una bebida hecha a base de lentejas y cebada, que recibe el nombre de

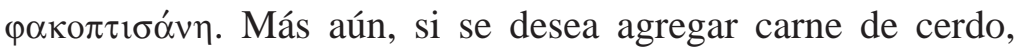
continúa diciendo Galeno, la que está fresca le va mejor a la tisana (recordemos que Apicio añade un codillo de cerdo a la preparación), mientras que la que está conservada con sal resulta más apropiada para combinarla con las lentejas (pero de éstas dos, la que se acomoda mejor para la

\footnotetext{
${ }^{45}$ Apic., IV, IV, 2 (174), TISANAM BARRICAM: infundes cicer, lenticulam, pisam. Defricas tisanam cum leguminibus elixas. Vbi bene bullierit, olei satis mittis et super uiridia concidis porrum, coriandrum, anetum, feniculum, betam, maluam, coliculum mollem; haec uiridia minuta concisa in caccabum mittis. Coliculos elixas et teres feniculi semen satis, origanum, silfi, ligusticum. Postquam triueris, liquamine temperabis et super legumina refundis et agites. Coliculorum minutas super concidis.

${ }^{46}$ Gal., VI, 526-527 K.
} 
aquella que recién ha sido salada, nuper salsam). Esta receta es útil para engrosar los humores, para incrementar la sangre cuando es débil, y para aumentar la producción de bilis negra; además posee la virtud de combinar dos extremos: el poder laxante de la cebada, las lentejas y las acelgas, con la facultad astringente del puerro. Por último, Galeno — quien no quiere que el médico ignore el arte culinaria $-{ }^{47}$ señala que puede agregarse ajedrea y poleo a la $\varphi \alpha \kappa o \pi \tau \imath \sigma \alpha ́ v \eta$, y puerro y anís a la tisana, para que, así preparados, estos alimentos resulten no sólo más fáciles de digerir, sino más agradables al paladar; ${ }^{48}$ aquí, cabe hacer notar que acelga, ajedrea, poleo, puerro y anís también figuran entre los ingredientes de las recetas apicianas citadas antes.

En suma, en las recetas médicas contenidas en De re coquinaria, se advierte que siguen, en cierta medida, las prescripciones galénicas, pero que también se desvían de ellas, atendiendo a las costumbres del pueblo; de hecho, Celso, médico romano de época de Tiberio, ya había advertido que "no hay que evitar ninguna clase de alimento del que se sirve el pueblo". ${ }^{49}$ Tampoco debe olvidarse que el manual apiciano estaba destinado a los cocineros y al público en general, no a los médicos, y por ello era indispensable que una obra de esta naturaleza proporcionara algunos remedios útiles y fáciles de preparar en casa, a los cuales recurrir en primera instancia.

El recetario de Apicio, indudablemente recoge parte significativa del saber tradicional de su tiempo en cuestión de alimentos, en materia no sólo de culinaria, sino también de medicina, si bien sobre este tema todavía resta mucho por estudiar. Las recetas médicas del De re coquinaria ponen de manifiesto, una vez más, la diversidad de fuentes de las que

\footnotetext{
${ }^{47}$ Gal., VI, 609 K.

${ }^{48}$ Gal., VI, $528 \mathrm{~K}$.

${ }^{49}$ Cels., I, I, 2: nullum genus cibi fugere, quo populus utatur.
} 
abreva el manual apiciano, pues con seguridad varias de esas recetas fueron extraídas de los distintos tratados o compendios que en materia de medicina circulaban en su época.

Una vez más se alcanza a vislumbrar la enorme riqueza contenida en las páginas del De re coquinaria, pues, como espero haber mostrado, esta obra incluye no sólo recetas sofisticadas y exóticas destinadas a una clase social opulenta y, en opinión de algunos, hasta cierto punto depravada, como se ha querido ver, sino recetas sencillas, de cuño popular, e incluso recetas saludables.

\section{BIBLIOGRAFÍA}

André, Jacques, Apicius, L'art culinaire, text. ét., trad. et comm., Paris, "Les Belles Lettres" (Collection des Universités de France, Guillaume Budé), 1987 (1a. ed. 1974), XXXII + 234 págs.

—, L'Alimentation et la cuisine à Rome, Paris, "Les Belles Lettres”, 1981, 2a. ed., 252 págs.

CRAIK, Elizabeth, "Hippokratic Diaita", Food in Antiquity, John Wilkins, David Harvey and Mike Dobson (eds.), Exeter, University of Exeter Press, 1995, pp. 343-350.

García Ballester, Luis, Galeno. En la sociedad y en la ciencia de su tiempo, Madrid, Guadarrama, 1972, 291 págs.

García Gual, Carlos, "Dieta hipocrática y prescripciones alimenticias de los pitagóricos", Dieta mediterránea. Comidas y hábitos alimenticios en las culturas mediterráneas, Aurelio Pérez Jiménez y Gonzalo Cruz Andreotti (eds.), Madrid, Ediciones Clásicas (Mediterranea, 6), 2000, pp. 43-67.

García Soler, María José, El arte de comer en la antigua Grecia, Madrid, Biblioteca Nueva, 2001, 462 págs.

Gil Sotres, Pedro, "Les régimes de santé", Histoire de la pensée médicale en Occident, t. 1: Antiquité et Moyen Age, Mirko D. Grmek (dir.), Paris, Éditions du Seuil, 1993, pp. 257-281.

Gourevitch, Danielle, "Les voies de la connaissance: la médicine dans le monde romain", Histoire de la pensée médicale en Occident, t. 1: Antiquité et Moyen Age, Mirko D. Grmek (dir.), Paris, Éditions du Seuil, 1993, pp. 94-122. 
Grant, Mark, Galen on Food and Diet, London-New York, Routledge, 2000, 214 págs.

Lejavitzer, Amalia, "Garum paradoxum, misterio y maravilla de la cocina romana", Noua tellus, 18-2, 2000, pp. 115-128.

MazzinI, Innocenzo, La medicina dei Greci e dei Romani. Letteratura, lengua, scienza, 2 vols., Roma, Jouvence, 1997.

Milham, Mary Ella (ed.), Apicius, Decem libri qui dicuntur De re coquinaria et Excerpta a Vinidario conscripta, Leipzig, Teubner (Bibliotheca Scriptorum Graecorum et Romanorum Teubneriana), 1969, XVI + 116 págs.

Nutton, Vivian, "Galen and the Traveller's Fare", Food in Antiquity, John Wilkins, David Harvey and Mike Dobson (eds.), Exeter, University of Exeter Press, 1995, pp. 359-370.

Powell, Owen, Galen, On the Properties of Foodstuffs (De alimentorum facultatibus), intr., trans. and comm., Cambridge, University Press, 2003, 206 págs.

Volpe Cacciatore, Paola, "El régimen según Galeno", Dieta mediterránea. Comidas y hábitos alimenticios en las culturas mediterráneas, Aurelio Pérez Jiménez y Gonzalo Cruz Andreotti (eds.), Madrid, Ediciones Clásicas (Mediterranea, 6), 2000, pp. 91-101. 